\title{
Autopercepción de los estudiantes acerca de la adquisición de las CTs y su contribución a la mejora de las actividades de aprendizaje
}

\section{Juan Antonio Llorens-Molina}

${ }^{a}$ E.T.S de Ingeniería Agronómica y del Medio Natural. Grupo IEMA, ICE Universitat Politècnica de València. E-mail : juallom2@qim.upv.es

\begin{abstract}
The self-perception of students about the acquisition of transversal competences can provide valuable guidelines for the design and assessment of learning activities, if considered these as the central axis of teaching planning. This paper presents the results of a survey carried out over two consecutive years, in which it has been tried to assess the degree to which students consider that some transversal competences are being developed from a certain activity. This has consisted in the elaboration through teamwork of screencasts based on a study of the composition and properties of foods of vegetable origin from the chemical point of view. The results have shown that this self-perception provides useful criteria to improve and adapt learning activities to the acquisition of some transversal competencies. Likewise, there are activities whose potential in this concern exceeds the limits of the control points, which would allows a more profound and effective assessment of the transversal competencies.
\end{abstract}

Keywords: Transversal competencies, assessment, self-perception, metacognition, screencasts, chemistry, food technology.

\footnotetext{
Resumen

La autopercepción de los estudiantes acerca de la adquisición de las competencias transversales puede proporcionar orientaciones valiosas para el diseño y evaluación de las actividades de aprendizaje, considerando éstas como el eje central de la planificación docente. En este trabajo se presentan los resultados de una encuesta realizada a lo largo de dos cursos consecutivos, en las que se ha evaluado el grado en que los estudiantes consideran que determinadas competencias transversales están siendo desarrolladas a partir de una determinada actividad. Ésta ha consistido en la elaboración mediante trabajo en equipo de screencasts basados en el estudio desde el punto de vista químico, de la composición y propiedades de alimentos de origen vegetal. Los resultados han evidenciado que dicha autopercepción proporciona criterios útiles para mejorar y adecuar las actividades de aprendizaje a la adquisición de determinadas competencias. Asimismo, es notorio también el hecho de que determinadas actividades poseen una potencialidad en este sentido que excede los límites de los puntos
} 
de control, lo que permitiría una evaluación más profunda y efectiva de las CTs.

http://inred.blogs.upv.es/

Palabras clave: Competencias trasnversales, evaluación, autopercepción, metacognición, screencasts, química, tecnología de alimentos.

\section{Introducción}

A lo largo de los últimos años se han prodigado numerosas y útiles propuestas en el marco del proyecto de Competencias Transversales de la Universidad Politécnica de Valéncia, (Poyecto Competencias Transversales UPV, 2015) tal como queda bien patente en las recientes ediciones de INRED. En este contexto de concreción y puesta en práctica de iniciativas para el desarrollo y evaluación de dichas competencias, el presente trabajo pretende incidir, desde esta óptica, en la evaluación de las actividades de aprendizaje.

Esta contribución parte de la consideración de las actividades, en cuanto a su naturaleza, diseño, secuenciación y evaluación como el eje de la planificación docente (Penzo, 2010). Desde este punto de vista es importante destacar el carácter dinámico y adaptativo que como consecuencia de la irrupción de las nuevas tecnologías ha adquirido su diseño. En efecto, la concepción estática, de las actividades de aprendizaje, característica del libro de texto y de los proyectos educativos tradicionales, puede actualmente considerarse superada. Los recursos tecnológicos de que ahora disponemos nos permiten adaptar cualquier actividad a los contextos tan diversos y cambiantes con que nos enfrentamos. Por otra parte, su desarrollo puede apoyarse en información ad hoc, tanto textual como audiovisual mediante instrumentos relativamente sencillos y asequibles. Si a ello añadimos la facilidad con que las nuevas tecnologías permiten crear ambientes de aprendizaje interactivos y colaborativos, puede afirmarse que el concepto de actividad de aprendizaje ha experimentado un cambio radical en los últimos años (Maceiras et al., 2010). Una importante consecuencia es el papel que adquiere la evaluación de las propias actividades en la planificación de la docencia, en sus diferentes niveles de concreción. Esta faceta no sería más que uno de los múltiples aspectos de la evaluación concebida, no como un proceso paralelo o complementario de la actividad docente, sino intrínsecamente ligado a la misma en sus múltiples aspectos (Boud y Douchy, 2010). Así, podemos formularnos, entre otras, las siguientes preguntas acerca del diseño de las actividades de aprendizaje:

- Forman parte de una secuencia estructurada? ¿Cuál es su papel en dicha secuencia? ¿Se tiene presente que dicha secuencia se enmarca, junto con otras, en el desarrollo de un curriculum aplicado en un contexto institucional concreto?

- ¿QQué procesos cognitivos entran en juego? ¿De qué recursos dispone el estudiante para abordarla?

- Tiene en cuenta la múltiple cantidad de aprendizajes generados por la vida cotidiana y el medio cultural del estudiante, al margen de las instituciones educativas? 
- ¿¿En qué grado producen el aprendizaje previsto? ¿Qué competencias entran en juego, tanto específicas como transversales? ¿Percibe conscientemente el estudiante que dichas competencias están siendo desarrolladas?

\section{Objetivos}

Si nos centramos en la última de las cuestiones planteadas, ha de considerarse el papel de la metacognición en el aprendizaje (Veenman et al., 2006). Este es un aspecto esencial del mismo. En efecto, la autopercepción por el estudiante de sus logros es un requisito para que un aprendizaje pueda considerarse como tal. Este proceso ha sido ampliamente estudiado en relación a la adquisición de las competencias transversales (Gomez-Puertas et al., 2014; Sanz et al., 2015). El objetivo de este trabajo se sitúa dentro de esta perspectiva y es evaluar el grado en que los estudiantes perciben que determinadas CTs están siendo puestas en juego en una determinada actividad. La actividad seleccionada ha sido la elaboración de un screencast por los estudiantes en torno a la naturaleza química y propiedades de determinados alimentos. Las CTs que a priori se han considerado involucradas en esta actividad han sido: Conocimiento y comprensión, trabajo en equipo y liderazgo, responsabilidad ética, medioambiental y profesional, comunicación efectiva, pensamiento crítico, planificación y gestión del tiempo, conocimiento de problemas contemporáneos e instrumental específica, siendo las dos últimas, puntos de control de la asignatura.

\section{Desarrollo de la innovación}

\section{1 ¿Por qué un screencast?}

Recordemos que el screencast consiste en la producción de un video a partir de la captura de pantalla de una secuencia de imágenes, acompañadas frecuentemente de la correspondiente grabación en audio, que puede ser creada, por ejemplo, a partir de una presentación de diapositivas.

¿Por qué se ha elegido el screencast? Por varias razones: en primer lugar, porque integra procesos comunicativos diversos: imágenes y textos orales y escritos; en segundo lugar, tal como se describirá más adelante, por su gran versatilidad en cuanto a su función en el proceso de aprendizaje; por último, porque puede crearse fácilmente mediante herramientas de acceso gratuito que proporcionan productos audiovisuales de razonable calidad sin necesidad de una gran experiencia previa en su uso.

El screencast es un recurso ampliamente utilizado tanto en la enseñanza online como material de apoyo a la clase presencial y sobre el que existe una amplia literatura relacionada con su diseño y modo de aplicación (Aguirre et al., 2012; Mohorovičić, 2012; Smith, 2014). La gran mayoría de investigaciones relacionadas con su utilización docente se refieren a material producido por el profesorado con diferentes finalidades. Sin embargo, otra importante contribución del screencast a la mejora de los procesos de aprendizaje es su elaboración por los estudiantes, con diversos objetivos. Diferentes autores coinciden en considerar esta herramienta como un formato idóneo para la realización de actividades que impliquen procesos de indagación y de relación entre conceptos. De este modo, constituyen 
también un buen punto de partida para la evaluación formativa. A partir de la información obtenida a través de los screencasts producidos por los estudiantes, puede proporcionarse una retroalimentación adecuada que favorezca la actividad metacognitiva del estudiante y la superación de errores conceptuales (Haxton y McGarvey, 2011; Croft et al., 2013; Soto y Ambrose, 2016)

Otra opción, a la que correspondería la actividad estudiada en este trabajo, es la elaboración y sistematización de información por los estudiantes de modo que pueda ser compartida por el resto de alumnos del grupo. En el ámbito agroalimentario puede citarse el estudio realizado por Pérez (2018).

\subsection{Contexto y metodología}

\subsubsection{Alumnado participante}

Este estudio ha sido realizado en la asignatura Fundamentos Químicos para Ciencia y Tecnología de Alimentos, dentro de la unidad didáctica dedicada a la introducción de la química orgánica, en el grado de Ciencia y Tecnología de los Alimentos, en la Universitat Politècnica de València. Se ha desarrollado a lo largo de los dos últimos cursos participando en la actividad un total de 154 estudiantes, siendo 135 el número de encuestas procesadas (55 y 80 en cada uno de los cursos, respectivamente).

\subsubsection{Descripción de la actividad}

Su realización se plantea de modo voluntario, aunque en la práctica es llevada a cabo por la mayoría de los estudiantes que participan de modo regular en la asignatura (81.5 \% y 96.7 \%, sobre el número de estudiantes calificados en la unidad didáctica, en los cursos 2016-17 y 2017-18, respectivamente). Dicha actividad ha consistido en la elaboración de un screencast de una duración entre 5 y 10 minutos, cuyos objetivos fueron:

- Describir detalladamente la composición química de un alimento de origen vegetal

- Representar la estructura molecular de cinco de los principales componentes, mediante la fórmula de esqueleto y un modelo tridimensional, utilizando aplicaciones informáticas específicas (ChemDraw ${ }^{\circledR}$ o $\quad$ ChemSketch ${ }^{\circledR}$, por ejemplo)

- Especificar en dichas moléculas qué grupos funcionales estudiados en el curso aparecen.

- Relacionar algunas de las propiedades nutritivas o medicinales de dicho alimento con la presencia de determinados compuestos químicos. En algunos casos podrá ser conveniente proporcionar alguna información sobre su procesado o modo de consumo.

En cuanto al modo de llevar a cabo la actividad, se realizó en equipos de tres personas. En cada uno de ellos, uno de los miembros asume el rol de interlocutor con el profesor de cara a enviar y recibir información a través de la plataforma "PoliformaT" de la UPV. Cada grupo elabora una presentación en Power-point ${ }^{\circledR}$ (entre 6 y 8 diapositivas, incluyendo la inicial) según los objetivos anteriormente descritos sobre un alimento asignado por el profesor. A partir de dicha presentación se realiza un vídeo que incorpora grabación de voz 
mediante un programa de descarga gratuita. En la primera diapositiva debe aparecer el alimento estudiado con una imagen, el nombre de la asignatura indicando que se trata de la Unidad Didáctica 1, y nombre y apellidos de los tres integrantes del grupo, presentándose también cada uno a través de la grabación en audio. La última diapositiva debe incluir la bibliografía consultada, sin olvidar citar la procedencia de las ilustraciones que no sean originales. Por último los vídeos son alojados en la plataforma https://media.upv.es/ a la que todos los alumnos pueden acceder para evaluar los vídeos.

La coevaluación se realizó online por el profesor y por tres compañeros de otros grupos, según una distribución realizada aleatoriamente por el profesor y comunicada a través de la plataforma PoliformaT. Se llevó a cabo a través de una rúbrica que responde a los objetivos de la actividad anteriormente señalados, cuyo diseño y aplicación son descritos más detalladamente por Llorens-Molina y Llorens. (2018).

\subsubsection{Encuesta planteada}

La encuesta planteada se aplicó en forma de escala Likert de acuerdo con las siguientes categorías: 1. Completamente de acuerdo; 2. De acuerdo; 3. Indiferentes; 4. En desacuerdo, 5. Completamente en desacuerdo. Fue remitida y contestada online a través de "espacio compartido" de PoliformaT y se introdujo mediante una breve explicación acerca de sus objetivos. Cada competencia transversal considerada se explicó brevemente en el propio texto de la encuesta, tal como se indica en el apéndice, al final del trabajo.

\section{Resultados}

Los siguientes diagramas (Fig. 1-9) muestran los resultados de la encuesta en los cursos 2016/17 y 2017/18. Las instrucciones y el formato fueron los mismos en ambos. Tan solo cabe indicar, como elementos diferenciales, la aportación en el segundo curso de vídeos tutoriales ("screencasts") para facilitar el uso de la aplicación ChemSketch y la divulgación, a modo de referencia, de dos de los vídeos realizados el curso anterior donde el tratamiento de las dos competencias transversales que son punto de control fue mejor aplicado.

La gráfica de la figura 1 muestra los datos globales correspondientes a los dos cursos. A pesar del resultado generalmente satisfactorio registrado para todas las competencias transversales evaluadas, si se considera la suma de "indiferente", "en desacuerdo" y "totalmente en desacuerdo", hay tres que muestran un resultado relativamente desfavorable: "Responsabilidad ética, medioambiental y profesional" (43.0\%), "Conocimiento de problemas contemporáneos", que es punto de control en la asignatura (33.3 \%) y "Pensamiento crítico" (24.5\%). El desarrollo del resto de competencias es percibido de un modo ampliamente favorable, si se considera la suma de "Muy de acuerdo" y "De acuerdo". Destacan principalmente: "Comprensión e integración” (94.1 \%), "Trabajo en equipo y liderazgo" (93.3 \%) y "Comunicación efectiva” (90.4\%). Ninguna de las tres es punto de control; no obstante "Instrumental específica", que sí lo es, registra también una elevada consideración (86.7 \%), del mismo modo que "Planificación y gestión del tiempo" (86.0 \%). 
Autopercepción de los estudiantes acerca de la adquisición de las CTs y su contribución a la mejora de las actividades de aprendizaje

Si se analizan las diferencias entre los dos cursos (Figuras 2-9), son generalmente pequeñas aunque ligeramente crecientes, sobre todo si los resultados se interpretan considerando dos bloques: "Muy de acuerdo" y "De acuerdo", y el resto. Así, por ejemplo, para “Comprensión e integración” tendríamos un 92,7 \% y 95.0 \%, para 2016/17 y 2017/18, respectivamente, "Trabajo en equipo y liderazgo", $90.9 \%$ y $95.1 \%$; "Responsabilidad ética, medioambiental profesional”, 52.7 \% y 60.0 \%; “Comunicación efectiva” 89.1 \% y 91.3\%; "Pensamiento crítico” 74.5 y 76.3 \%, "Planificación y gestión del tiempo”, 90.9 y 82.5 \% (única competencia en cuyo desarrollo se observa una disminución de la percepción positiva). Son precisamente las dos competencias que son punto de control las que registran un mayor aumento de su valoración positiva, que en el caso de "Conocimiento de problemas contemporáneos" es notable (58.5 - $72.5 \%$ ). "Instrumental específica” aumenta desde un 81.8 a un $90.0 \%$. Dado que los únicos cambios en el diseño y planteamiento de la actividad afectan, como ya se indicó anteriormente, a estas dos competencias, podría atribuirse a ellos la evolución observada, siempre teniendo en cuenta, por supuesto, el alcance y validez de datos cualitativos como los obtenidos en esta encuesta.

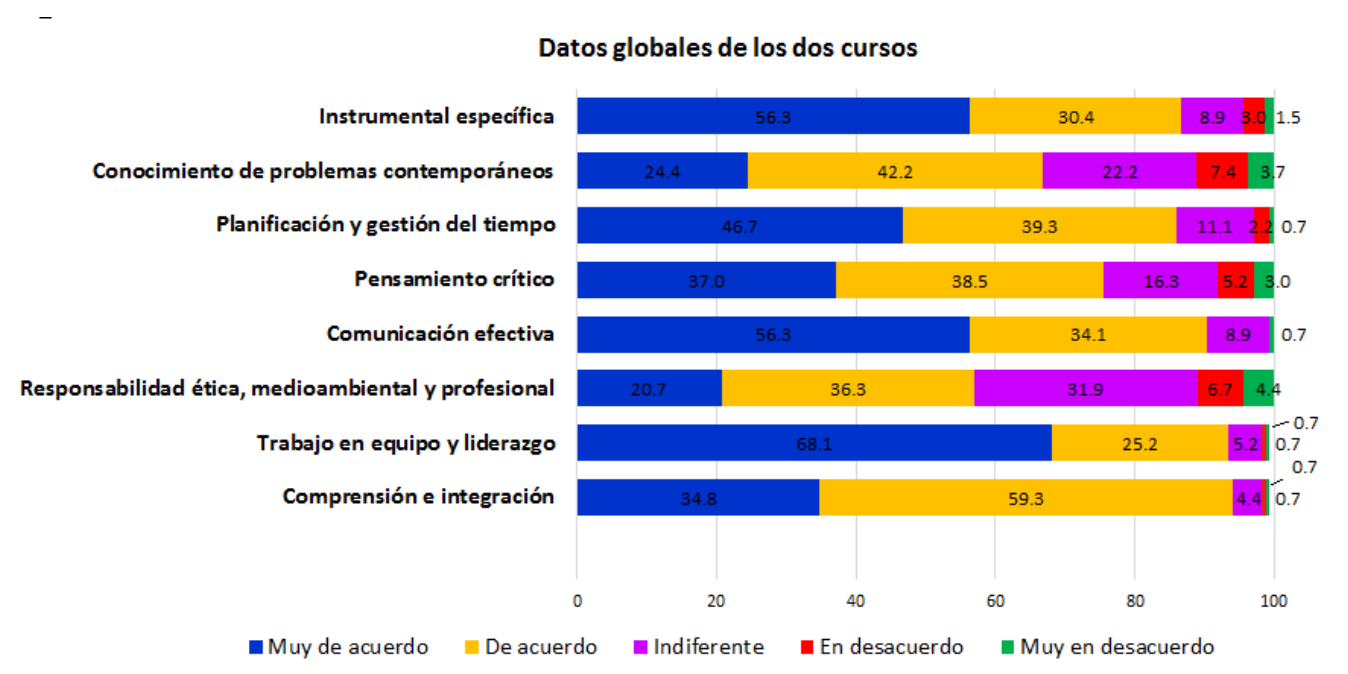

Fig. 1. Datos globales de los dos cursos para cada competencia transversal

\section{Comprensión e integración}

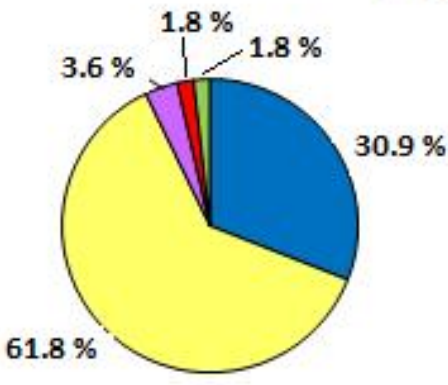

2016/17
Muy de acuerdo

$\square$ De acuerdo

$\square$ Indiferente

aEn desacuerdo

$\square$ Muy en desacuerdo

(cc) EY-NC-ND

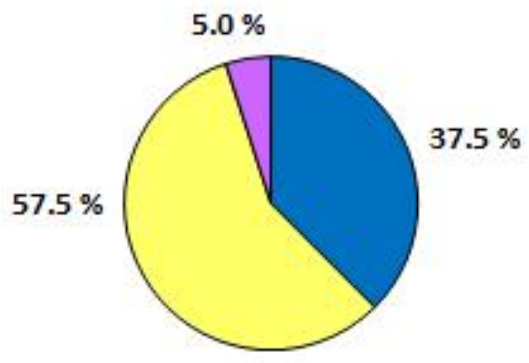

2017/18 
Fig. 2. Resultados de la encuesta relativos a la competencia transversal "Comprensión e integración"

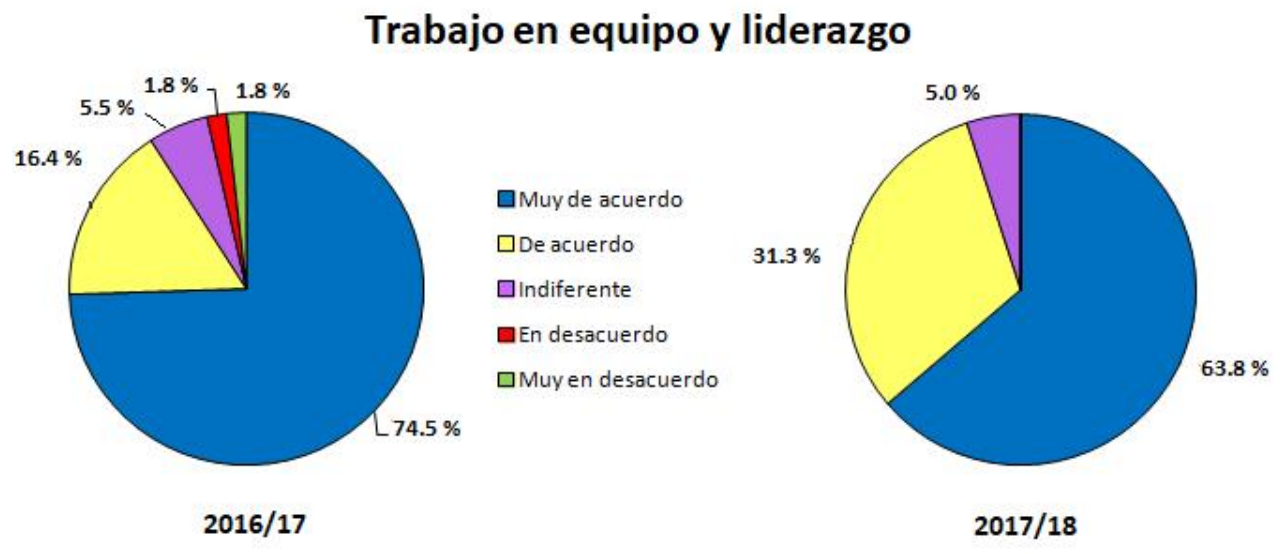

Fig. 3. Resultados de la encuesta relativos a la competencia transversal "Trabajo en equipo y liderazgo".

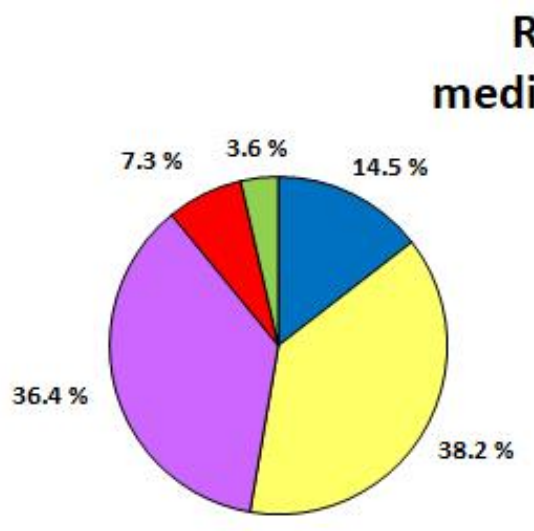

2016/17

\section{Responsabilidad ética,} medioambientaly profesional

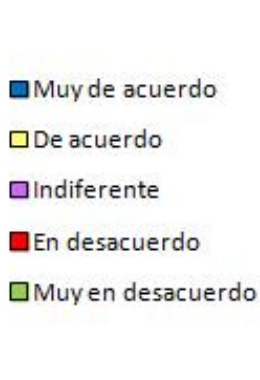

$\square$ Muy de acuerdo

$\square$ De acuerdo

口indiferente

En desacuerdo

$\square$ Muyen desacuerdo

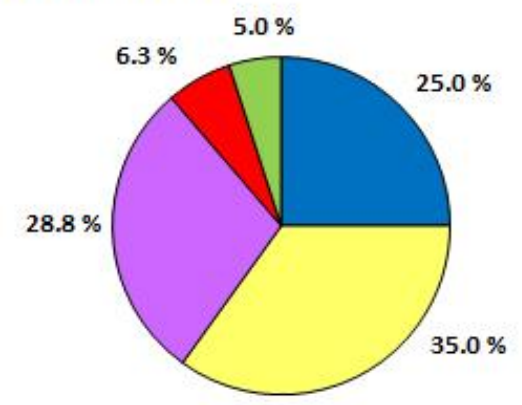

$2017 / 18$

Fig. 4. Resultados de la encuesta relativos a la competencia transversal "Responsabilidad ética, medioambiental y profesional"

2018, Universitat Politècnica de València

Congreso In-Red (2018) 


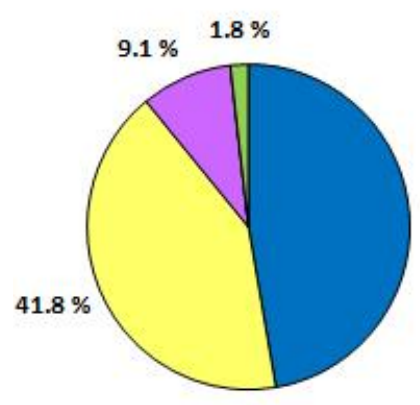

2016/17

\section{Comunicación efectiva}
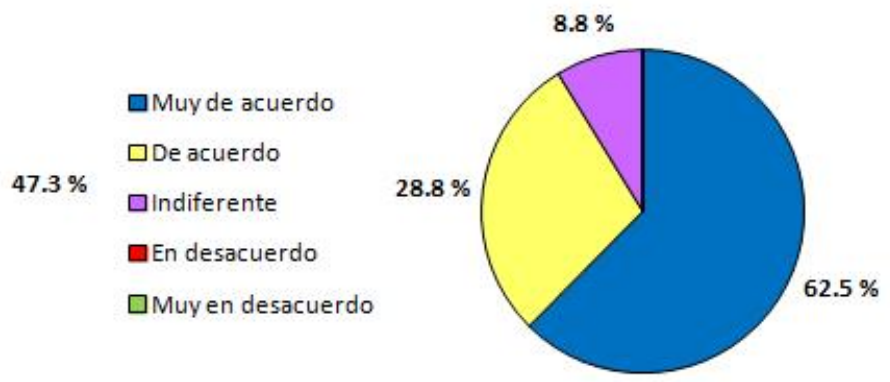

$2017 / 18$

Fig. 5. Resultados de la encuesta relativos a la competencia transversal "Comunicación efectiva"

\section{Pensamiento crítico}

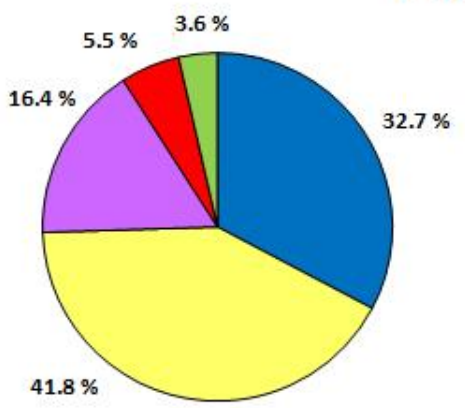

$2016 / 17$

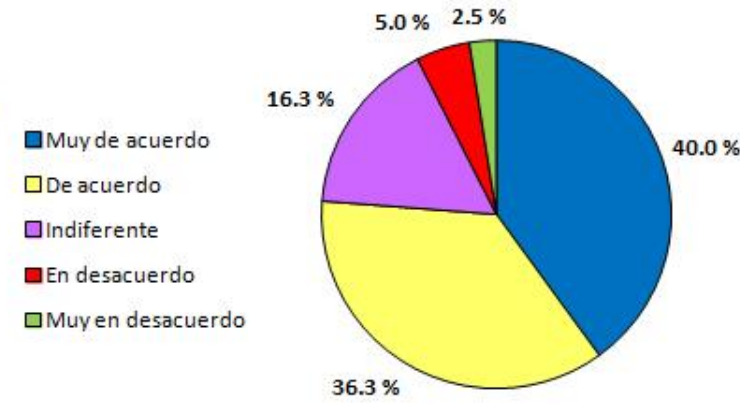

$2017 / 18$

Fig. 6. Resultados de la encuesta relativos a la competencia transversal "Pensamiento crítico"

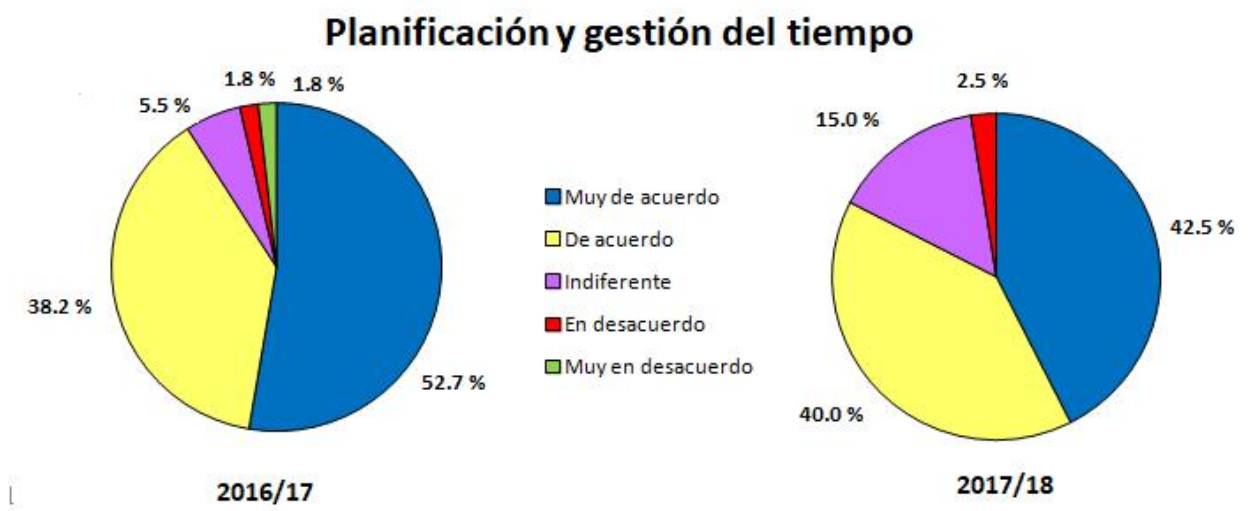

Fig. 7. Resultados de la encuesta relativos a la competencia transversal "Planificación y gestión del tiempo". 


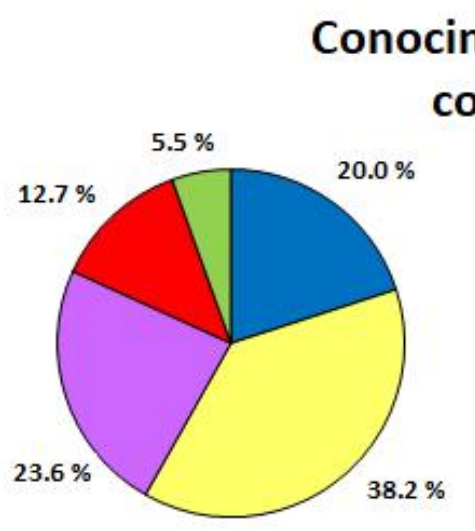

$2016 / 17$

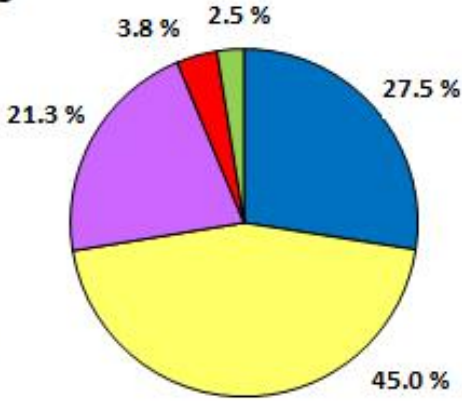

$2017 / 18$

$45.0 \%$

Fig. 8. Resultados de la encuesta relativos a la competencia transversal "Conocimeinto de problemas contemporáneos"

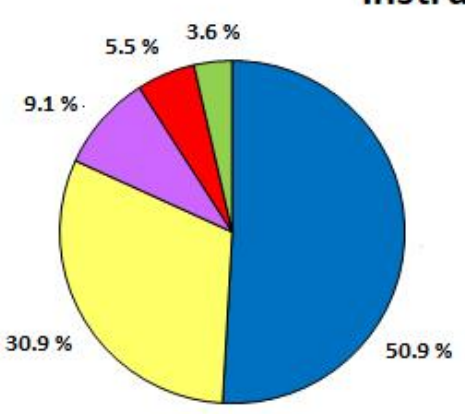

2016/17
๑Muyde acuerdo

口De acuerdo

口Indiferente

En desacuerdo

$\square$ Muy en desacuerdo

Fig. 9. Resultados de la encuesta relativos a la competencia transversal "Instrumental específica"

\section{Conclusiones}

Desde una planificación educativa centrada en el diseño y evaluación de las actividades de aprendizaje, cabría cuestionar el concepto de "punto de control" en la evaluación de las competencias transversales. Por una parte, es evidente que su asignación a cada asignatura permite asegurar su desarrollo y evaluación a lo largo del grado y en el conjunto de las distintas materias impartidas; no obstante, la evidencia de que determinadas actividades también contribuyen, tal vez en mayor grado, a la adquisición de otras que no son punto de control, llevaría a considerar la conveniencia de ser también evaluadas. La propuesta concreta sería que, una vez establecidas las competencias que son punto de control, se pudiera incluir la evaluación de otras cuyo desarrollo, a través de las actividades de la asignatura, resulte evidente y sea percibido por los estudiantes.

2018, Universitat Politècnica de València

Congreso In-Red (2018) 
Evaluar la percepción de los estudiantes muestra puede ser, por tanto, una práctica útil en la medida en que constituye un criterio para considerar qué competencias transversales cabe evaluar en una asignatura y proporciona un feed-back apropiado para mejorar el diseño de las actividades. Al margen de las dudas que pueda generar el hecho de una amplia aceptación de la actividad descrita en este trabajo (que puede deberse a una satisfacción general con la asignatura o el profesorado), aparecen diferencias lo suficientemente notorias como para replantear aspectos de su diseño y gestión. Es evidente, por ejemplo, que la actividad planteada adolece de escaso estímulo hacia un enfoque basado en la consideración de valores éticos, desde un punto un punto de vista crítico. Una contextualización más explícita de los objetivos de la actividad en problemas de interés tales como el de las distintas opciones surgidas en la sociedad frente a la alimentación, también podrían mejorar el tratamiento de la competencia «conocimiento de problemas contemporáneos », que es punto de control.

\section{Referencias}

AGUIRRE, S., BARRA, E., QUEMADA, J., MENDIOLA, M. A., PASTOR, J. Y., MARTINEZ, M. E. y PORTAENCASA, R. (2012). “Comunica-Media. Uso de la grabación de clases, el screencast y la videoconferencia en el aula“, Revista del Congrés Internacional de Docència Universitària i Innovació (CIDUI), 1(1), http://www.cidui.org/revistacidui/index.php/cidui/article/viewFile/396/390, (Consulta: 12 de marzo de 2018)

BOUD, D., y DOCHY, F. (2010). “Assessment 2020. Seven propositions for assessment reform in higher education“.

https://lirias.kuleuven.be/bitstream/123456789/263461/2/Assessment-2020_propositions_final.pdf (Consulta: 12 de marzo de 2018)

CARR, A. y LY, P. (2009). "More than words": screencasting as a reference tool“, Reference Services Review, vol. 37, issue 4, p. 408-420.

CROFT, T., DUAH, F., y LOCH, B. (2013). “I'm worried about the correctness': undergraduate students as producers of screencasts of mathematical explanations for their peers-lecturer and student perceptions“, International Journal of Mathematical Education in Science and Technology, vol. 44, issue 7, p. 1045-1055.

GOMEZ-PUERTAS, L., ROCA-CUBERES, C. y GUERRERO-SOLE, F. (2014). “¿Cómo perciben los estudiantes la adquisición de competencias? Análisis comparado: Teorías de la Comunicación en la Universidad Pompeu Fabra“, Historia y Comunicación Social, vol. 19, special issue february, p. 313-326.

HAXTON, K. J. y MCGARVEY, D. J. (2011). "Screencasting as a means of providing timely, general feedback on assessment", New Directions in the Teaching of Physical Sciences, vol.7, p. 1821.

LLORENS-MOLINA J. A. y LLORENS DE JAIME, J. (2018), "Screencasts in the Classroom. Design and Assessment“. INTED, 2018

LLORENS-MOLINA, J. A. (2018), "Introductory Organic Chemistry For Food Science And Technology“, INTED 2018.

MACEIRAS, R., CANCELA, Á. Y GOYANES, V. (2010). “Aplicación de nuevas tecnologías en la docencia universitaria », Formación universitaria, vol. 3, issue 1, p. 21-26.

MOHOROVICIC, S. (2012), «Creation and use of screencasts in higher education». In MIPRO, 2012 Proceedings of the 35th International Convention, p. 1293-1298.

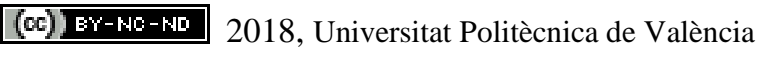

Congreso IN-RED (2018) 
PENZO, W., FERNANDEZ, V., GARCIA, I., GROS, B., PAGES, T., ROCA, M., Y VENDRELL, P. (2010). «Guía para la elaboración de las actividades de aprendizaje» Cuadernos de docencia universitaria, vol. 15, p. 1-67.

Pérez, S., Funciones de los Objetos de Aprendizaje en la Enseñanza de la Química de los Alimentos. FUNCIONES DE LOS OBJETOS DE APRENDIZAJE EN LA ENSEÑANZA DE LA QUÍMICA DE LOS ALIMENTOS. http://conhisremi.iuttol.edu.ve/articles.php?code=PUBL000018

PROYECTO INSTITUCIONAL DE COMPETENCIAS TRANSVERSALES (2015), Universitat Politècnica de València, https://www.upv.es/entidades/ICE/info/U0724624.pdf (Consulta: 12 de marzo de 2018)

SMITH, P. (2014). "Screencasting as a means of Enhancing the Student Learning Experience ».Learning and Teaching in Action, http://www.celt.mmu.ac.uk/ltia/Vol10Iss1/7 smith.pdf (Consulta: 12 de marzo de 2018).

SOTO, M. y AMBROSE, R. (2016). «Screencasts: Formative assessment for mathematical thinking », Technology, Knowledge and Learning, vol. 21, issue 2, p. 277-283.

VEENMAN, M. V., VAN HOUT-WOLTERS, B. H., y AFFLERBACH, P. (2006). « Metacognition and learning: Conceptual and methodological considerations » Metacognition and learning, vol.1, issue 1, p. 3-14. 


\section{Apéndice}

\section{ENCUESTA SOBRE COMPETENCIAS TRANSVERSALES (Presentación y texto explicativo de cada una)}

Como sabes, a partir de este curso se evalúan las competencias transversales. La actividad que has realizado sobre la composición química de los alimentos tiene como objetivo contribuir a su desarrollo y se ha introducido a nivel experimental. Por eso ha sido voluntaria y solamente contribuye a la calificación aumentándola.

Nos parece importante conocer vuestra opinión acerca de cómo esta actividad puede contribuir al logro de algunas de estas competencias. Para ello hemos preparado la siguiente encuesta. En ella verás una serie de competencias con una breve descripción de las mismas y una escala de valoración. Se trata simplemente de marcar con una cruz el recuadro correspondiente.

\section{COMPRENSIÓN E INTEGRACIÓN}

Comprender quiere decir "percibir y tener una idea clara de lo que se dice, se hace o sucede o descubrir el sentido profundo de algo". Para demostrar que algo se ha comprendido, la persona identifica y recupera la información y la explica con sus propias palabras, interpretando e integrando las ideas desde su propia perspectiva.

\section{TRABAJO EN EQUIPO Y LIDERAZGO}

El trabajo en equipo implica crear y desarrollar un clima de confianza mutua entre los componentes que permita trabajar de forma responsable y cooperativa. El término más apropiado para describir esta situación es COMPARTIR: compartir conocimientos, compromiso y responsabilidad.

\section{RESPONSABILIDAD ÉTICA, MEDIOAMBIENTAL Y PROFESIONAL}

Esta competencia se refiere al conjunto de conocimientos, habilidades, destrezas y actitudes, útiles para interactuar con el entorno, de forma ética, responsable y sostenible.

\section{COMUNICACIÓN EFECTIVA}

Comunicarse efectivamente significa tener desarrollada la capacidad de transmitir conocimientos, ideas y argumentos de manera clara y rigurosa, tanto de forma oral como escrita, con los recursos apropiados y adaptándose a las circunstancias y al tipo de público.

\section{PENSAMIENTO CRÍTICO}

El pensamiento crítico implica poner en cuestión nuestra forma habitual de pensar y actuar. Es el pensamiento de los interrogantes: ¿por qué las cosas son así?, ¿por qué las cosas no pueden ser de otro modo?, ¿por qué tú crees que son así?, etc.

\section{PLANIFICACIÓN Y GESTIÓN DEL TIEMPO}

Esta competencia implica ser capaz de organizar y distribuir correctamente el tiempo del que disponemos para alcanzar nuestros objetivos a corto, medio y largo plazo.

\section{CONOCIMIENTO DE PROBLEMAS CONTEMPORÁNEOS}

Esta competencia implica comprender las cuestiones y valores políticos, sociales, legales y medioambientales contemporáneos. Se trata de que desarrollen la capacidad de "estar al día" de los eventos actuales en su campo de conocimiento y en la sociedad en general. 


\section{INSTRUMENTAL ESPECÍFICA}

Esta competencia se refiere al uso de las herramientas y tecnologías necesarias para el ejercicio profesional asociado a cada titulación. El estudiante será capaz de identificar las herramientas más adecuadas en cada caso, conociendo sus utilidades y siendo capaz de integrarlas y combinarlas para poder resolver un problema, realizar un proyecto o un experimento.

(cc) EY-NC-ND 2018, Universitat Politècnica de València 